Radiologe 2018 · 58:312-319

https://doi.org/10.1007/s00117-018-0372-x

Online publiziert: 2. März 2018

(c) Der/die Autor(en) 2018. Dieser Artikel ist eine Open-Access-Publikation.

CrossMark
H. Schöllnast

Universitätsklinik für Radiologie, Medizinische Universität Graz, Graz, Österreich

\section{Radiologische Charakterisierung chronisch-entzündlicher Darmerkrankungen}
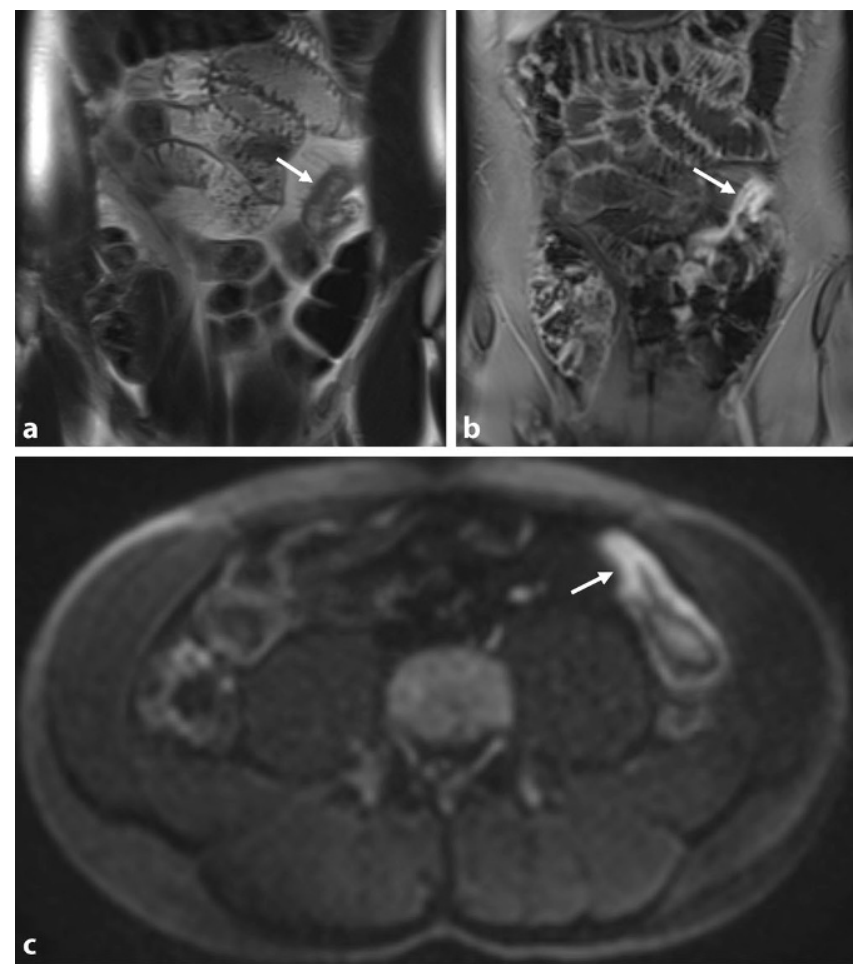

Abb. 1a-d 27-jähriger Patient mit aktivem inflammatorischem M. Crohn und segmentalem Befall des Jejunums (Pfeil). Die koronaren HASTE-Sequenzen (a) zeigen eine hyperintense Wandverdickung mit unscharfer Begrenzung. Die Wand nimmt verstärkt Kontrastmittel auf, wobei die Form

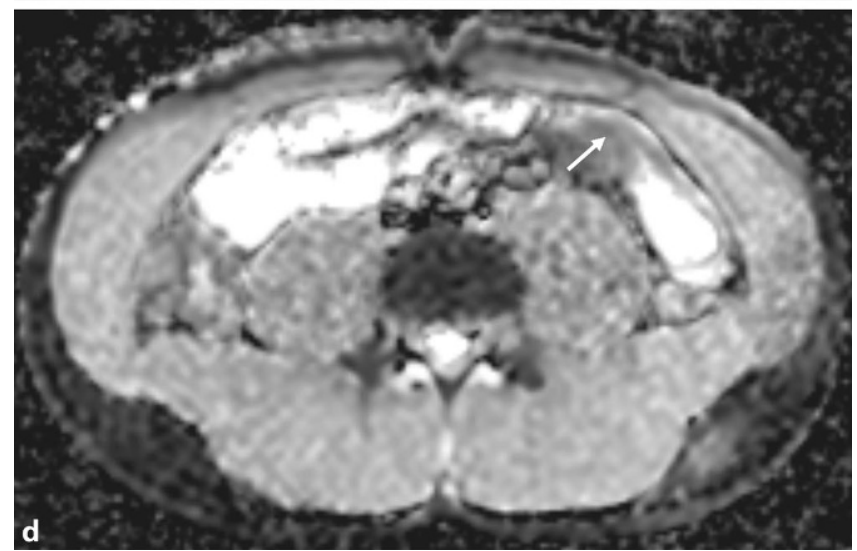
der Aufnahme geschichtet ist (b). In den diffusionsgewichteten Sequenzen (c) stellt sich die Darmwand hyperintens dar mit hypointensem Signal im ADC-Mapping (d). HASTE "half Fourier-acquired single-shot turbo-spin echo", $A D C$ „apparent diffusion coefficient" 

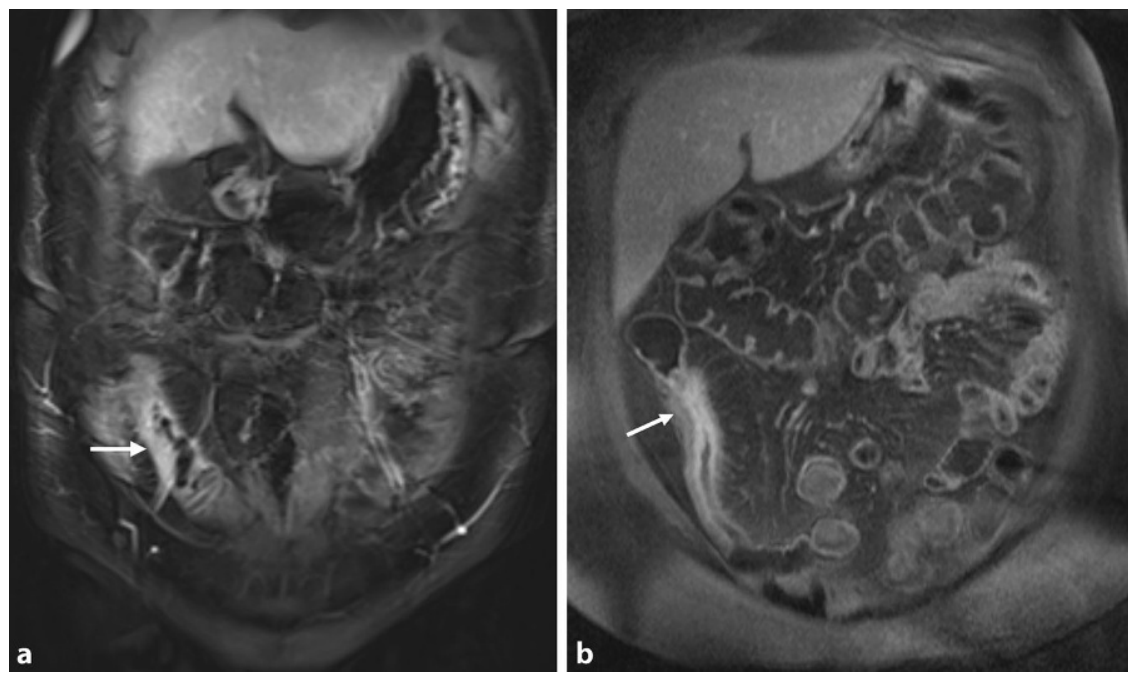

Abb. $2 \Delta$ a 71-jährige Patientin mit M. Crohn: Die Kontrastmittelaufnahme der Darmwand ist homogen, d. h. in allen Wandschichten gleich ausgeprägt. b 58-jähriger Patient mit M. Crohn: Die Kontrastmittelaufnahme ist in der Mukosa deutlich stärker ausgebildet als in den übrigen Wandschichten

postuliert, wobei es zu einer Immunreaktion gegen die Darmflora kommt [2]. Die Inzidenz wird in Deutschland und Österreich mit 6,6-11,5 pro 100.000 Einwohner angeben $[21,22]$. Es besteht keine wesentliche Geschlechtsprädominanz. Der mediane Altersgipfel liegt bei beiden Entitäten zwischen 20 und 30 Jahren, mit einem zweiten Gipfel zwischen 60 und 70 Jahren beim M. Crohn und 70-80 Jahren bei der Colitis ulcerosa. Prinzipiell kann die Erkrankung jedoch in jedem Lebensalter auftreten [2].

\section{Morbus Crohn}

Der M. Crohn ist eine chronisch rezidivierende entzündliche Erkrankung, die sich entlang des gesamten Gastrointestinaltrakts manifestieren kann. Die Ileozökalregion ist in ca. $50 \%$ der Fälle betroffen, gefolgt vom Ileum mit $30 \%$ und vom Kolon mit 20\% [2]. Der entzündliche Prozess ist transmural, d.h. die Darmwand ist in ihrergesamten Schichtung betroffen. Fistelbildungen sind daher häufige Komplikationen eines M. Crohn. Der Befall erfolgt diskontinuierlich, wodurch befallene und nichtbefallene Darmanteile aufeinander folgen können. Letztere werden als ,skip lesions“ bezeichnet. Der M. Crohn kann in 4 Subtypen eingeteilt werden:

1. aktiv inflammatorisch,

2. fistulierend/perforierend,

\section{MR-Enterographie}

Die Magnetresonanztomographie(MRT) ist aufgrund der fehlenden Strahlenexposition die bevorzugte bildgebende Methode in der Evaluierung einer chronisch-entzündlichen Darmerkrankung. In einer rezenten Metaanalyse wurde berichtete, dass MR-Enterographie, MREnteroklysma und CT-Enterographie eine vergleichbare Genauigkeit in der Detektion eines M. Crohn aufweisen [13]. Die MR-Enterographie und das MR-Enteroklysma ermöglichen jedoch eine Beurteilung der Aktivität bzw. der Subtypen der Erkrankung [13]. Die Gefahr einer inkompletten Füllung ist bei der MR-Enterographie höher als beim MR-Enteroklysma [3, 20]. Bei der Beurteilung von Patienten mit M. Crohn wurden jedoch keine signifikanten Unterschiede zwischen beiden Füllungsmethoden gefunden, wenngleich oberflächliche Pathologien besser mit dem MREnteroklysma abgrenzbar waren [17, 20, 25]. In den meisten Zentren hat sich aufgrund des Patientenkomforts die MREnterographie durchgesetzt. Indikationen zur Durchführung sind:

- Beurteilung der Lokalisation der entzündlichen Veränderungen und deren Ausdehnung,

- Klassifikation der Subtypen,

- Verlaufskontrolle bei bekannter Erkrankung,

- Ausschluss einer Erkrankung bei entsprechenden Symptomen,

- suszipiertes Rezidiv,

- Monitoring des Therapieansprechens,

- Planung eines chirurgischen Eingriffs $[10,16]$.

sigmoid betroffen, eine Ausdehnung auf das gesamte Kolon und in schweren Fällen bis in das terminale Ileum ist jedoch möglich [9]. Die Entzündung ist im Gegensatz zum M. Crohn im Regelfall auf die Mukosa und Submukosa beschränkt und geht nicht in tiefere Schichten der Darmwand über. In schweren Fällen kann eine transmurale Ausbreitung beobachtet werden [9]. In einigen Fällen sind beide Entitäten nicht zu differenzieren. Diese Fälle werden als unklassifizierbare CED bezeichnet.
Um eine Distension des Darmlumens und einen hohen Kontrast zwischen Darmlumen und Darmwand zu erhalten, werden enterale Kontrastmittel verabreicht. Am weitesten verbreitet ist die Verwendung von biphasischen enteralen Kontrastmitteln, welche sich hypointens in $\mathrm{T} 1$ und hyperintens in $\mathrm{T} 2$ darstellen $[11,18,26]$. Das hypointense Signal in den T1-gewichteten Sequenzen verbessert den Kontrast zwischen dem Darmlumen und der vermehrt kontrastmittelaufnehmenden entzündeten Darmwand, das hyperintense Signal in 
den T2-gewichteten Sequenzen ermöglicht die Darstellung kleiner Ulzera, die sich in die hypointense Darmwand erstrecken $[18,30]$. Polyethylenglykol weist einen exzellenten Kontrast auf und führt aufgrund seiner hohen Osmolarität zur Darmdistension $[1,18,19]$. Andere Kontrastmittel beinhalten Mannitol, Sorbitol oder Laktulose [28].

Als Vorbereitung zur MR-Enterographie fasten die Patienten 4-6h vor der Untersuchung. Ein Volumen von $1000-1500 \mathrm{ml}$ biphasischen Kontrastmittels wird oral verabreicht [18, 28]. Die Transitzeit bis zum Kolon beträgt in den meisten Fällen 40-60 min, kann jedoch - insbesondere nach Teilresektionen des Dünndarms - wesentlich kürzer sein. Mittels dickschichtigen, T2-gewichteten MRCP-Sequenzen (Magnetresonanzcholangiopankreatikographie) können der Transit und der Grad der Distension festgestellt werden. Antiperistaltische Medikamente wie HyoscinN-butylbromid (Buscopan ${ }^{\circledR}$ ) oder Glukagon $\left(\right.$ GlucaGen $\left.^{\circledR}\right)$ werden intravenös injiziert, um die Peristaltik und die Bewegungsartefakte $\mathrm{zu}$ reduzieren [31]. Hyoscin-N-butylbromid $\quad\left(\right.$ Buscopan $^{\circledR}$ ) wird als Mittel der 1. Wahl empfohlen [28].

Folgende MR-Sequenzen werden empfohlen [28]:

- axiale und koronare, 2-D, „fast-

spin echo“, T2-gewichtete Sequenzen

(z.B. HASTE [„half Fourier-acquired single-shot turbo-spin echo“], SSFSE ["single-shot fast-spin echo"], SSTSE ["single-shot turbo-spin echo“]) ohne Fettsättigung ( $\leq 5 \mathrm{~mm}$ Schichtdicke),

- axiale und koronare, 2-D, „steadystate-free-precession“ Gradientenecho-Sequenzen (z. B. TrueFISP [,true fast imaging with steady precession“], FIESTA [„fast imaging employing steady-state acquisition"], T2-FFE [„fast-field echo"]) ohne Fettsättigung ( $\leq 5 \mathrm{~mm}$ Schichtdicke),

- axiale oder koronare „fast-spin echo“ T2-gewichtete Sequenzen mit Fettsättigung ( $\leq 5 \mathrm{~mm})$,

- koronare, 3-D, T1-gewichtete Sequenzen mit Fettsättigung vor Kontrastmittelgabe (z. B. VIBE [,volumetric interpolated breath hold examination“], LAVA [„,iver acqui-

Radiologe 2018 · 58:312-319 https://doi.org/10.1007/s00117-018-0372-x

๑ Der/die Autor(en) 2018. Dieser Artikel ist eine Open-Access-Publikation.

\section{H. Schöllnast}

\section{Radiologische Charakterisierung chronisch-entzündlicher Darmerkrankungen}

\section{Zusammenfassung}

Hintergrund. Chronisch-entzündliche Darmerkrankungen (CED) zeigen im Krankheitsverlauf unterschiedliche Aktivität. Diese beeinflusst neben den morphologischen Veränderungen maßgeblich die Wahl der Therapie.

Fragestellung. Die Möglichkeiten der Bildgebung in der Charakterisierung der Aktivität entzündlicher Veränderungen werden evaluiert.

Material und Methoden. Das vorliegende Review untersucht die aktuelle Literatur zu Indikationen, Durchführung und Befunden der Bildgebung chronisch-entzündlicher Darmerkrankungen und zur Beurteilung von deren Aktivität.
Ergebnisse. Die magnetresonanztomographische (MR-)Enterographie erlaubt die Beurteilung der Aktivität der entzündlichen Darmerkrankung und ermöglicht eine Subtypisierung.

Schlussfolgerung. Die MR-Enterographie liefert neben Klinik, Labor und Endoskopie wesentliche Informationen für die Einschätzung der Aktivität einer CED.

Schlüsselwörter

Morbus Crohn · Colitis ulcerosa · Krankheitsaktivität · Magnetresonanz-Enterographie . Klassifikation

\section{Radiological characterization of chronic inflammatory bowel disease}

\section{Abstract}

Background. During the course of the disease, chronic inflammatory bowel diseases (CIBD) show different activities, which-in addition to morphological changes-significantly influence the choice of therapy.

Objective. The value of imaging in characterization of the activity of inflammatory changes is evaluated.

Materials and methods. In the present review of the current literature, indications, performance, and findings of imaging of inflammatory bowel disease and on the assessment of disease activity are assessed.
Results. Magnetic resonance (MR) enterography allows the assessment of inflammatory bowel disease activity and subtyping. Conclusion. In addition to clinical, laboratory, and endoscopic information, MR enterography provides essential information for the assessment of CIBD activity.

\section{Keywords}

Crohn disease - Ulcerative colitis - Disease activity - Magnetic resonance enterography . Classification sition with volume acceleration"], THRIVE [„T1-weighted high resolution isotropic volume examination"]),

- koronare und axiale, 3-D, T1-gewichtete Sequenzen $30 \mathrm{~s}$, $45 \mathrm{~s}$ und $70 \mathrm{~s}$ nach Kontrastmittelgabe $(\leq 3 \mathrm{~mm}$ Schichtdicke).

Das Gadolinium-haltige Kontrastmittel wird mit einer Dosis von 0,1-0,2 mmol pro Kilogramm Körpergewicht und einer Injektionsrate von $2 \mathrm{ml} / \mathrm{s}$ verabreicht, gefolgt von $20 \mathrm{ml} \mathrm{Kochsalzlösung} \mathrm{[28].}$ Axiale diffusionsgewichtete Sequenzen (b = 0-50, b =600-900, $5 \mathrm{~mm}$ Schichtdicke) und Cine-Sequenzen können additiv durchgeführt werden [28]. Derzeit gibt es keinen allgemein akzeptierten ADCSchwellenwert (,apparent diffusion coefficient") für die Differenzierung zwischen aktivem und inaktivem M. Crohn, da sowohl ödematöse als auch fibrotische Segmente eine Diffusionseinschränkung zeigen. Diffusionsgewichtete Bilder können aber in der Detektion entzündeter Darmabschnitte hilfreich sein [5].

\section{Befunde beim M. Crohn}

\section{Aktiver inflammatorischer Subtyp}

In der aktiven Phase kommt es zu einer zellulären Infiltration der Mukosa 

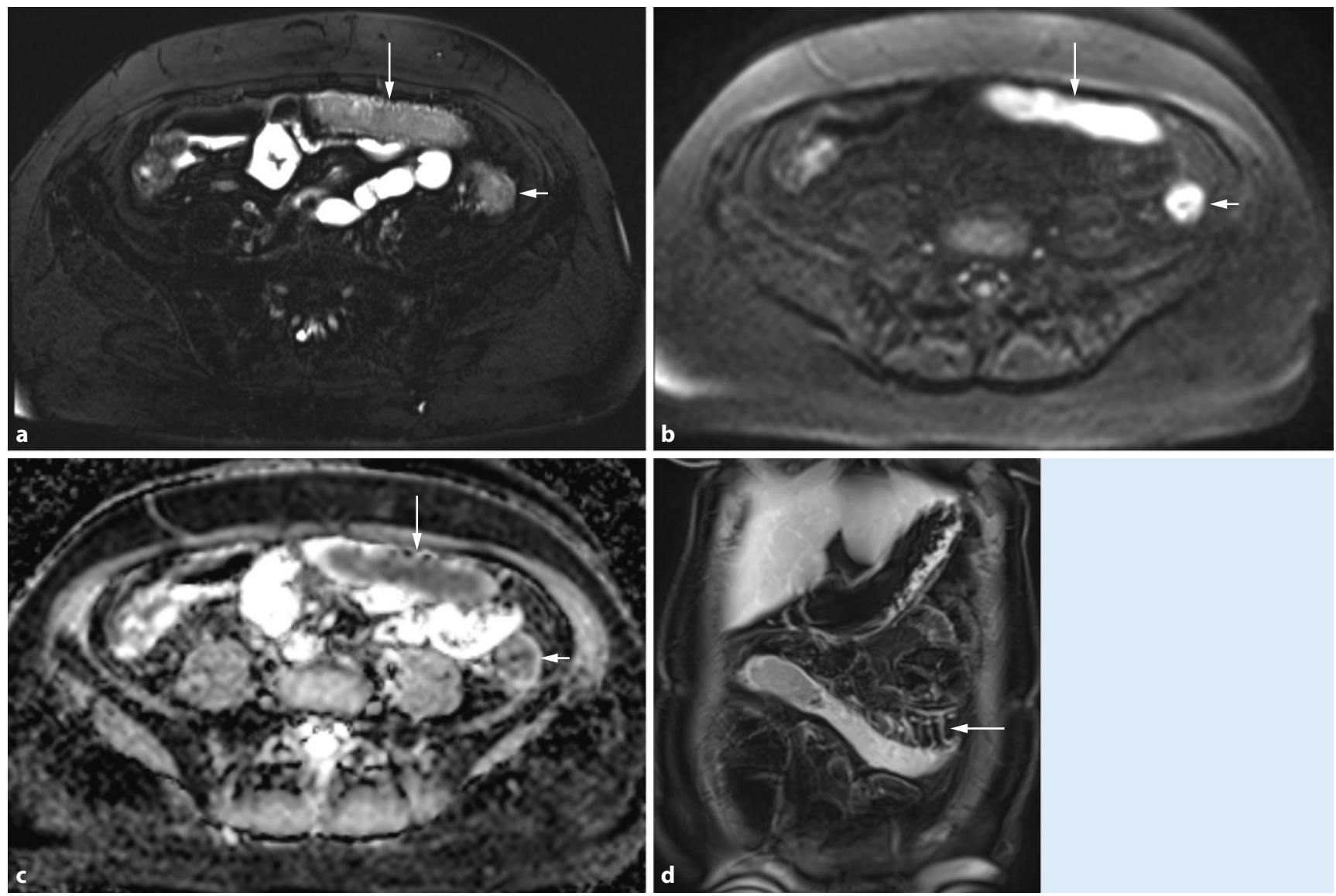

Abb. 3 ॥ 53-jährige Patientin mit M. Crohn und Befall des Colon transversum (langer Pfeil, a-c) und des Colon descendens ( $k$ urzer Pfeil, a-c). Die axialen HASTE-Sequenzen mit Fettunterdrückung (a) zeigen eine Lumeneinengung des Colon ascendens und descendens mit Ödem der Darmwand. Die diffusionsgewichteten Sequenzen (b) und das ADC-Mapping (c) bestätigen die akute Entzündung. $\mathbf{d}$ In den koronaren, kontrastmittelunterstützen VIBE-Sequenzen nimmt das Colon transversum verstärkt auf, wobei im angrenzenden Fettgewebe dilatierte Gefäße zur Darstellung kommen, die einen kammähnlichen

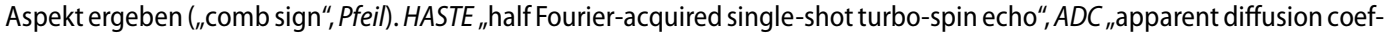
ficient", VIBE "volumetric interpolated breath hold examination"

mit Ulzerationen und Ödemzonen, was der Mukosa einen Kopfsteinpflaster-artigen Aspekt verleiht (,cobblestone pattern“; [2]). Das entzündliche Wandödem führt zu einer Verdickung der Darmwand (Dünndarm: $\geq 3 \mathrm{~mm}$, Dickdarm: $\geq 4 \mathrm{~mm}$, adäquate Distension vorausgesetzt), zu einem hyperintensen Signal der Darmwand in den T2-gewichteten Sequenzen, zu einer starken Kontrastmittelaufnahme der Darmwand in den T1-gewichteten, fettsupprimierten Sequenzen und einer eingeschränkten Diffusion der Darmwand in den diffusionsgewichteten Sequenzen ([27]; - Abb. 1). Wenn True-FISP-Sequenzen im Untersuchungsprotokoll inkludiert sind, sollte die Messung der Wanddicke bevorzugt in den HASTE-Sequenzen oder den fettsupprimierten, T1-gewich- teten Sequenzen durchgeführt werden, da die True-FISP-Sequenzen eine exakte Messung wegen der Chemical-ShiftArtefakte erschweren [16].

$\mathrm{Ob}$ eine Korrelation zwischen dem Grad der Kontrastmittelaufnahme und dem Grad der Entzündung vorliegt, ist derzeit unklar [9]. Es konnte jedoch gezeigt werden, dass das Muster der Kontrastmittelaufnahme mit dem Grad der Entzündung in der Histologie korreliert, wobei 3 Muster unterschieden werden [23]. Beim geschichteten Muster bzw. Zielscheibenmuster (,target sign") zeigt die Darmwand eine starke Kontrastmittelaufnahme der Mukosa und der Muskularis/Serosa mit deutlich geringerer bis fehlender Kontrastmittelaufnahme der dazwischen gelegenen ödematösen Submukosa ([4, 24]; - Abb. 1). Dieses
Muster korreliert mit dem höchsten Grad der Entzündung. Beim homogenen oder transmuralen Muster zeigt die gesamte Darmwand eine gleichmäßige Kontrastmittelaufnahme. Dieses Muster korreliert mit dem geringsten Grad der Entzündung. Beim mukosalen Muster ist die Kontrastmittelaufnahme auf die $\mathrm{Mu}$ kosa und Submukosa beschränkt. Dieses Muster kann als intermediär angesehen werden ([4, 24] - Abb. 2). Die ödematöse Wandverdickung kann zu einer Stenose führen, wobei diese von einer fibrostenosierenden Form angegrenzt werden muss, da erstere medikamentös und letztere chirurgisch behandelt wird.

Neben diesen Veränderungen der Darmwand können beim aktiven inflammatorischen Subtyp auch mesenteriale Veränderungen nachweisbar sein, 

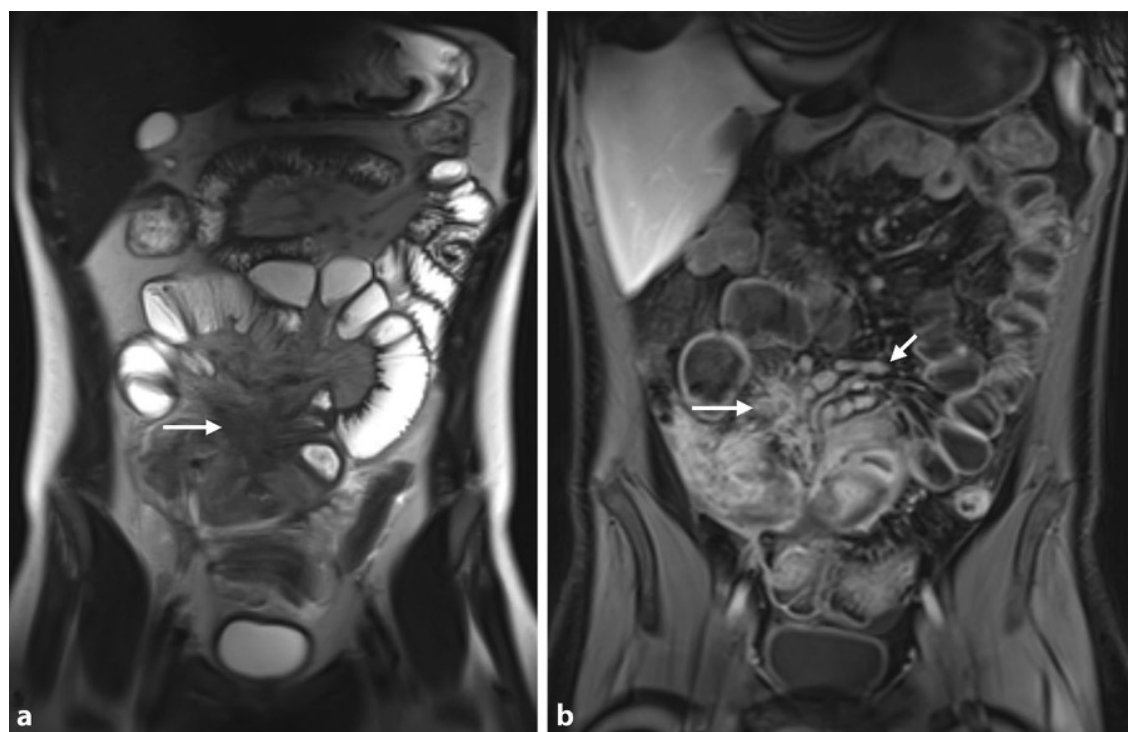

Abb. 4 ॥ 22-jähriger Patient mit M. Crohn und lleumbefall. In den koronaren HASTE-Sequenzen (a) ist eine sternförmige Fibrose des Mesenteriums nachweisbar (Pfeil), die in den späten kontrastmittelunterstützen Sequenzen (b) eine Kontrastmittelaufnahme zeigt (langerPfeil). Entlang der vaskulären Strukturen kommen multiple reaktive Lymphknoten zur Darstellung (kurzer Pfeil in b). HASTE "half Fourier-acquired single-shot turbo-spin echo"

welche aus einem Ödem und Gefäßdilatationen bestehen, wobei die dilatierten Gefäße den Aspekt eines Kammes ergeben („,comb sign“; 0 Abb.3). Eine reaktive Lymphadenopathie kann ebenfalls im Mesenterium gefunden werden. Für gewöhnlich sprechen Lymphknoten mit einem Querdurchmesser von $<1 \mathrm{~cm}$ für reaktive Lymphknoten. Bei einer Vergrößerung $>1 \mathrm{~cm}$ sollte an ein Malignom des Darms (Karzinom oder Lymphom) gedacht und die Art der Darmwandverdickung sorgfältig betrachtet werden $[6,8,9]$. Eine fibrös-fettige Proliferation des Mesenteriums führt durch die Fettgewebsvermehrung zu einer Separation der Dünndarmschlingen, welche wie in Fettgewebe eingepackt imponieren können. Wenn auch nicht limitiert auf den aktiven inflammatorischen Subtyp, so verstärkt die fibrös-fettige Proliferation bei diesem Subtyp den Aspekt des „, comb signs" [9].

\section{Fistulierender/perforierender Subtyp}

Die transmurale Entzündung und die tiefen Ulzerationen können die Serosa durchbrechen, wodurch Sinusgänge („,blind endend“) und Fisteln (Verbindungen mit anderen epithelialisierten Oberflächen - Organe oder Haut) ent- stehen $[9,27]$. Aus Sinusgängen und Fisteln können sich Abszesse bilden. In der MR-Enterographie stellen sich Sinusgänge und Fistel als tubuläre, in T2-gewichteten Sequenzen hyperintense Strukturen dar, die in den kontrastmittelunterstützten, fettsupprimierten, T1-gewichteten Sequenzen eine randständige Kontrastmittelaufnahme zeigen. Durch die entzündlichen Läsionen kann eine Fibrose des angrenzenden Mesenteriums resultieren, die meist eine sternförmige Form aufweist (• Abb. 4). Ein Abszess stellt sich als eine Flüssigkeitsformation mit komplexem Inhalt in T1- und T2gewichteten Sequenzen dar, welche eine randständige Kontrastmittelaufnahme zeigt.

\section{Fibrostenosierender Subtyp}

Die chronische Entzündung führt $\mathrm{zu}$ einer transmuralen Fibrose und zu einer Verdickung der Darmwand. Es resultieren Einengungen des Lumens und Strikturen, was zu Obstruktionen führen kann. In der MR-Enterographie ist das betroffene fibrotische Segment wandverdickt mit hypointensem Signal in den T2-gewichteten Sequenzen (• Abb. 5). Es zeigt sich typischerweise keine eingeschränkte Diffusion. Die Kontrastmittelaufnahme ist geringer ausgeprägt als beim aktiven inflammatorischen Subtyp und meist auf die Mukosa beschränkt. Die geschichtete und homogene Kontrastmittelaufnahme sind seltener [9, 23]. Bei Verwendung später kontrastmittelunterstützter Sequenzen kann eine protrahierte Kontrastmittelaufnahme beobachtet werden [24]. Eine Dilatation der vorgeschalteten Darmanteile gibt Hinweis auf die obstruktive Wirkung der Fibrose. Differenzialdiagnostisch ist eine Obstruktion durch postoperative Adhäsionen (Bridenileus) in Erwägung zu ziehen, wobei bei diesem ein abrupter, gewinkelter Übergang vom dilatierten in den kollabierten Darmanteil zu verzeichnen ist („,beak sign“), ohne Nachweis eines wandverdickten Segments in der Übergangszone. Aufgrund der akuten Klinik wird man mit einem Bridenileus bei Patienten mit M. Crohn eher im CT als in der MR-Enterographie konfrontiert sein, da in der akuten Situation meist ein CT durchgeführt wird. Da Stenosen sowohl im akuten als auch im chronischen Stadium auftreten können (im akuten Stadium durch eine reversible ödematöse Wandverdickung, im chronischen Stadium durch eine irreversible Fibrose), ist eine radiologische Einschätzung der Art der Stenose (Ödem vs. Fibrose) wesentlich für das weitere Management. Während aktive Läsionen medikamentös behandelt werden, ist bei fibrostenosierenden Läsionen ein chirurgisches Vorgehen notwendig [12]. Die mechanische Wirksamkeit einer Stenose kann neben der Beurteilung des Dilatationsgrades der vorgeschalteten Darmanteile mit Hilfe von CineSequenzen eingeschätzt werden [27].

\section{Reparativer/regenerativer Subtyp}

Bei diesem Subtyp fehlen sowohl aktive inflammatorische als auch fibrosierende Vorgänge. Es findet sich eine Atrophie der Mukosa mit regenerativen Polypen [15]. In der Submukosa kommt es zu Fettgewebseinlagerungen, was zu einer Schichtung in den T2-gewichteten Sequenzen führt. Fettunterdrückte Sequenzen führen zu einer Signalminderung und damit zu einer Differenzierung zu einem akuten Ödem $[14,16]$. 

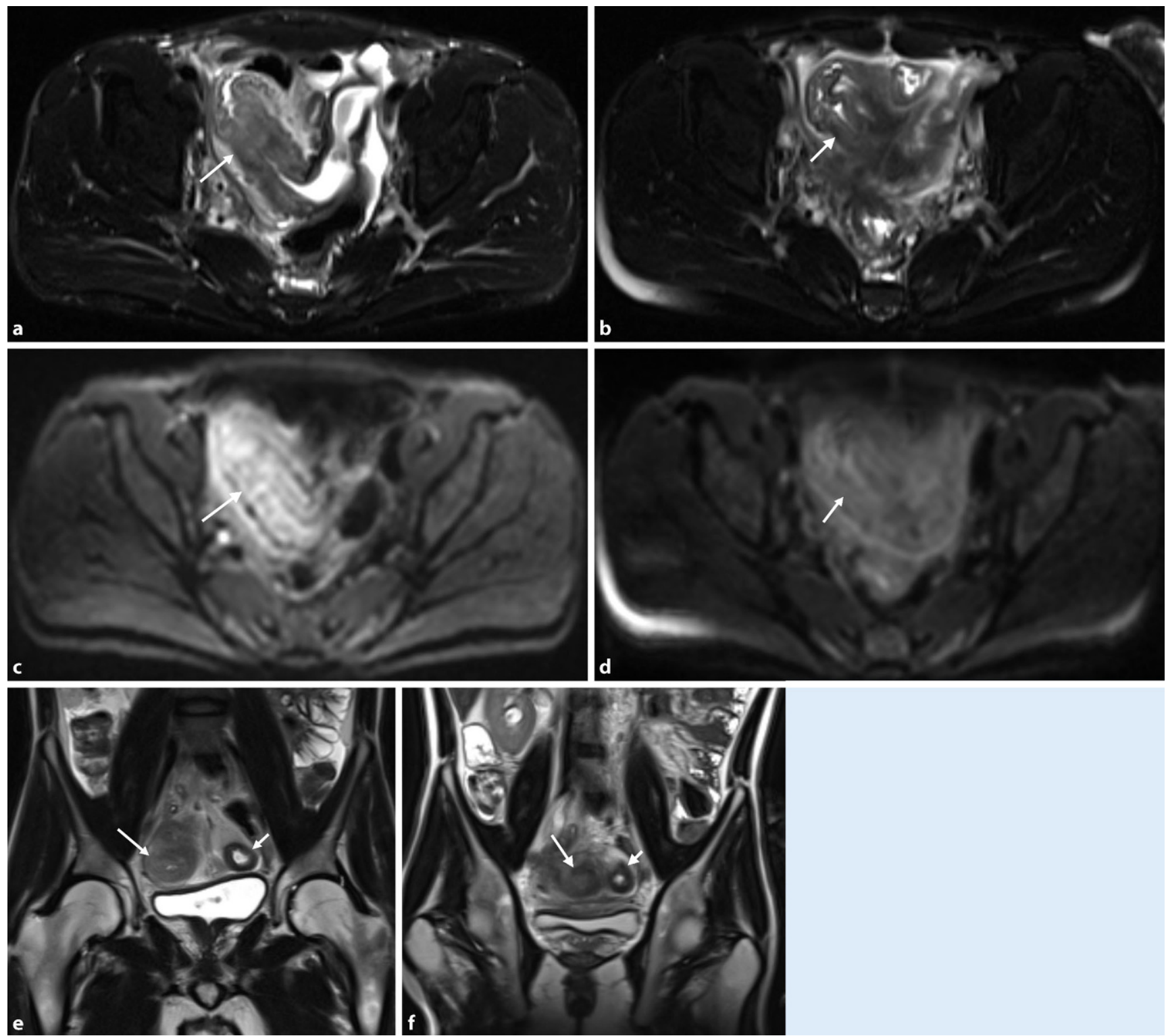

Abb. $5 \Delta$ 34-jähriger Patient mit aktivem M. Crohn (a, c, e) und Verlaufskontrolle nach 3 Monaten (b, d, f $)$. Die axialen, fettunterdrückten HASTE-Sequenzen (a, b) zeigen eine Abnahme des Wandödems. Auch die Diffusionseinschränkung hat abgenommen $(\mathbf{c}, \mathbf{d})$. In den koronaren HASTE-Sequenzen $(\mathbf{e}, \mathbf{f})$ gleicht sich das Wandsignal des betroffenen Segments (lange Pfeile) dem Wandsignal des nichtbetroffenen Segments (kurzePfeile) an. HASTE "half Fourier-acquired single-shot turbo-spin echo"

\section{Befunde bei Colitis ulcerosa}

\section{Akute Phase}

Die akuten entzündlichen Veränderungen der Colitis ulcerosa haben die Tendenz zu konfluieren, wobei scharfe Übergänge zwischen den betroffenen und nichtbetroffenen Darmabschnitten bestehen. In der Frühphase kommt es zu einem mukosalen Ödem mit konsekutiver Abstumpfung der Haustren, zu Granulationen und Hyperämie [7, 9]. Bei anhaltender Entzündung finden sich Einblutungen in die Mukosa und ober- flächliche Ulzera, die im weiteren Verlauf konfluieren und sich in die Submukosa ausdehnen können. Die zwischen den Ulzera ausgesparten Mukosainseln werden als inflammatorische Pseudopolypen bezeichnet [9]. In der MR-Enterographie ist im frühen Stadium lediglich die Hyperämie anhand einer verstärkten Kontrastmittelaufnahme der Darmwand darstellbar. Bei zunehmendem Ödem kommt es zu einer Wandverdickung mit einem hyperintensen Signal der Submukosa in den T2-gewichteten Sequenzen. Die inflammatorischen Pseudopolypen können bei hoher Auflösung abgebildet werden [9].

\section{Subakute und chronische Phase}

Das fortgeschrittene Studium ist von einem Verlust der Haustren, einer Wandverdickung und einer Verkürzung des Darms gekennzeichnet, was durch eine neuronale Hypertrophie und fibromuskulären Hyperplasie der Muscularis mucosae zustande kommt. Eine submuköse Fetteinlagerung und Fibrose trägt ebenfalls zur Wandverdickung bei $[7,9]$. In der MR-Enterographie zeigt 

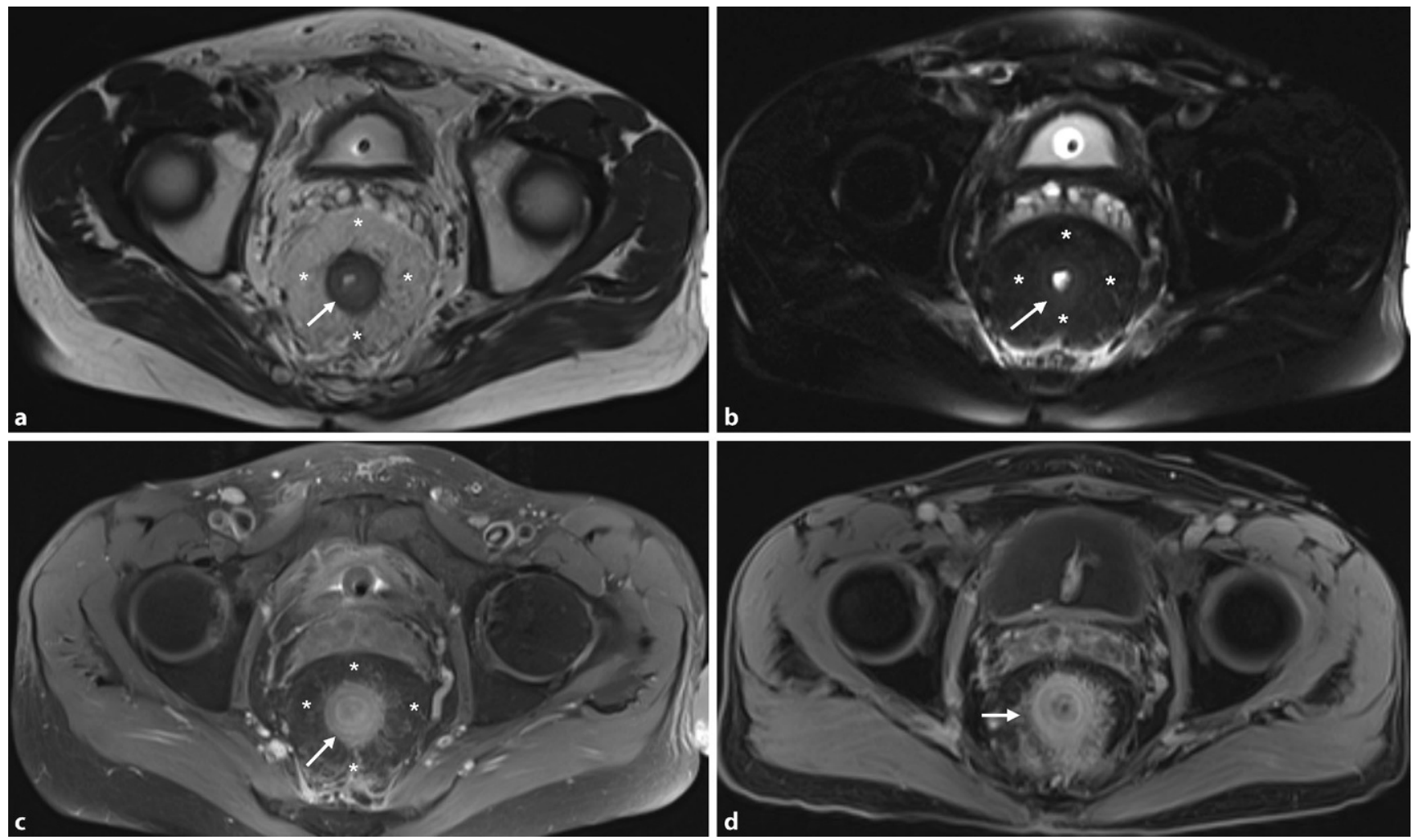

Abb. 6 - 56-jähriger Patient mit Colitis ulcerosa. In den HASTE-Sequenzen (a), fettsupprimierten HASTE-Sequenzen (b) und kontrastmittelunterstützten VIBE-Sequenzen (c) zeigt sich eine Vermehrung des präsakralen Fettgewebes (Sterne) mit Verlagerung der Samenblasen nach ventral. Es sind nur feine lineare Maskierungen des perirektalen Fettgewebes nachweisbar. In einer Kontrolluntersuchung nach 2 Monaten (d) zeigt sich eine zunehmende lineare Maskierung des perirektalen Fettgewebes (Pfeil) als Zeichen einer akuten Inflammation. HASTE "half Fourier-acquired single-shot turbo-spin echo", VIBE "volumetric interpolated breath hold examination"

die Wandverdickung daher ein leicht erhöhtes Signal in den T2-gewichteten Sequenzen. Postinflammatorische Pseudopolypen können abgrenzbar sein. Eine perirektale Fetteinlagerung ist ein charakteristisches Zeichen einer chronischen Colitis ulcerosa, welches in der MR-Enterographie durch eine Fettgewebsvermehrung im präsakralen Raum zur Darstellung kommt (• Abb. 6; [7]).

\section{Fazit für die Praxis}

- Chronisch-entzündliche Darmerkrankungen weisen im Krankheitsverlauf unterschiedliche Aktivität auf und sind durch Subtypen charakterisiert.

- Die MR-Enterographie erlaubt eine Beurteilung der Aktivität der Erkrankung und eine Klassifikation der Subtypen.

- Neben Klinik, Labor und Endoskopie ist die MR-Enterographie eine wichtige Methode in der Entschei- dungsfindung für das adäquate Patientenmanagement.

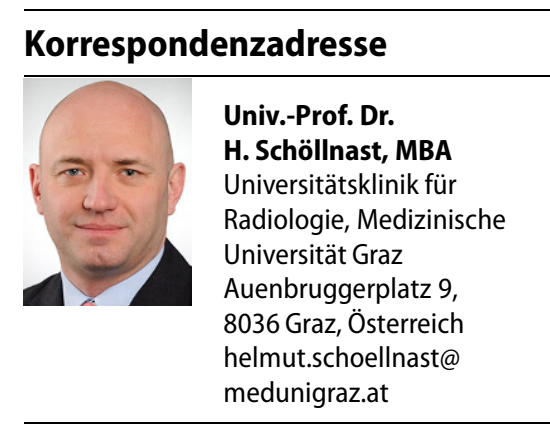

Funding. Open access funding provided by Medical University of Graz.

\section{Einhaltung ethischer Richtlinien}

Interessenkonflikt. H. Schöllnast gibt an, dass kein Interessenkonflikt besteht.

Dieser Beitrag beinhaltet keine vom Autor durchgeführten Studien an Menschen oder Tieren.
Open Access. Dieser Artikel wird unter der Creative Commons Namensnennung 4.0 International Lizenz (http://creativecommons.org/licenses/by/4.0/deed. de) veröffentlicht, welche die Nutzung, Vervielfältigung, Bearbeitung, Verbreitung und Wiedergabe in jeglichem Medium und Format erlaubt, sofern Sie den/die ursprünglichen Autor(en) und die Quelle ordnungsgemäß nennen, einen Linkzur Creative Commons Lizenz beifügen und angeben, ob Änderungen vorgenommen wurden.

\section{Literatur}

1. Ajaj W, Goyen M, Schneemann H et al (2005) Oral contrast agents for small bowel distension in MRI: influence of the osmolarity for small bowel distention. Eur Radiol 15:1400-1406

2. BaumgartDC, Sandborn WJ (2012)Crohn's disease. Lancet 380:1590-1605

3. Born C, Nagel B, Leinsinger G et al (2003) MRI with oral filling in patients with chronic inflammatory bowel diseases. Radiologe 43:34-42

4. Del Vescovo R, Sansoni I, Caviglia R et al (2008) Dynamic contrast enhanced magnetic resonance imaging of the terminal ileum: differentiation of activity of Crohn's disease. Abdom Imaging 33:417-424

5. Dohan A, Taylor S, Hoeffel C et al (2016) Diffusionweighted MRI in Crohn's disease: current status 
and recommendations. J Magn Reson Imaging 44:1381-1396

6. Fidler JL, Guimaraes L, Einstein DM (2009) MR imaging of the small bowel. Radiographics 29:1811-1825

7. Gore RM, Balthazar EJ, Ghahremani GG et al (1996) CT features of ulcerative colitis and Crohn's disease. AJR Am J Roentgenol 167:3-15

8. Griffin N, Grant LA, Anderson S et al (2012) Small bowel MR enterography: problem solving in Crohn's disease. Insights Imaging 3:251-263

9. Kaushal P, Somwaru AS, Charabaty A et al (2017) MR Enterography of inflammatory bowel disease with endoscopic correlation. Radiographics 37:116-131

10. Kilcoyne A, Kaplan JL, Gee MS (2016) Inflammatory bowel disease imaging: current practice and future directions. World J Gastroenterol 22:917-932

11. Laghi A, Carbone I, Catalano C et al (2001) Polyethylene glycol solution as an oral contrast agent for MR imaging of the small bowel. AJR Am J Roentgenol 177:1333-1334

12. Lee SS, Kim AY, Yang SK et al (2009) Crohn disease of the small bowel: comparison of CT enterography, MR enterography, and smallbowel follow-through as diagnostic techniques. Radiology 251:751-761

13. Liu W, Liu J, Xiao W et al (2017) A diagnostic accuracy meta-analysis of CT and MRI for the evaluation of small bowel Crohn disease. Acad Radiol 24:1216-1225

14. Maccioni F, Viscido A, Broglia L et al (2000) Evaluation of Crohn disease activity with magnetic resonance imaging. Abdom Imaging 25:219-228

15. Maglinte DD, Gourtsoyiannis N, Rex D et al (2003) Classification of small bowel Crohn's subtypes based on multimodality imaging. Radiol Clin North Am 41:285-303

16. Mantarro A, Scalise P, Guidi E et al (2017) Magnetic resonance enterography in Crohn's disease: how we do it and common imaging findings. World J Radiol 9:46-54

17. Masselli G, Casciani E, Polettini E et al (2008) Comparison of MR enteroclysis with MR enterography and conventional enteroclysis in patients with Crohn's disease. Eur Radiol 18:438-447

18. MasselliG, Gualdi G (2012) MRimaging of the small bowel. Radiology 264:333-348

19. Mckenna DA, Roche CJ, Murphy JM et al (2006) Polyethylene glycol solution as an oral contrast agent for MRI of the small bowel in a patient population. Clin Radiol 61:966-970

20. Negaard A, Paulsen V, Sandvik L et al (2007) A prospectiverandomized comparison between two MRI studies of the small bowel in Crohn's disease, the oral contrast method and MR enteroclysis. Eur Radiol 17:2294-2301

21. Ott C, Obermeier F, Thieler S et al (2008) The incidence of inflammatory bowel disease in a rural region of Southern Germany: a prospective population-based study. Eur J Gastroenterol Hepatol 20:917-923

22. Petritsch W, Fuchs S, Berghold A et al (2013) Incidence of inflammatory bowel disease in the province of Styria, Austria, from 1997 to 2007: a population-based study. J Crohns Colitis 7:58-69

23. Punwani $S$, Rodriguez-Justo $M$, Bainbridge $A$ et al (2009) Mural inflammation in Crohn disease: location-matched histologic validation of MR imaging features. Radiology 252:712-720

24. Rimola J, Planell N, Rodriguez S et al (2015) Characterization of inflammation and fibrosis in Crohn's disease lesions by magnetic resonance imaging. Am J Gastroenterol 110:432-440
25. Scharitzer M, Ba-Ssalamah A (2015) Modern MRI of the small bowell. Radiologe 55:1067-1076

26. Sood RR, Joubert I, Franklin H et al (2002) Small bowel MRI: comparison of a polyethylene glycol preparation and water as oral contrast media. JMagn Reson Imaging 15:401-408

27. Stanley E, Moriarty HK, Cronin CG (2016) Advanced multimodality imaging of inflammatory bowel disease in 2015: an update. World J Radiol 8:571-580

28. Taylor SA,AvniF, Cronin CGetal (2017) Thefirstjoint ESGAR/ESPR consensus statement on the technica performance of cross-sectional small bowel and colonic imaging. Eur Radiol 27:2570-2582

29. Triester SL, Leighton JA, Leontiadis Gl et al (2006) A meta-analysis of the yield of capsule endoscopy compared to other diagnostic modalities in patients with non-stricturing small bowel Crohn's disease. Am J Gastroenterol 101:954-964

30. Umschaden HW, Gasser J (2003) MR enteroclysis. Radiol Clin North Am 41:231-248

31. Umschaden HW, Szolar D, Gasser J et al (2000) Small-bowel disease: comparison of MRenteroclysis images with conventional enteroclysis and surgical findings. Radiology 215:717-725
Möchten Sie einen Beitrag für Der Radiologe einreichen?

Wir freuen uns, dass Sie unsere Zeitschrift Der Radiologe mitgestalten möchten.

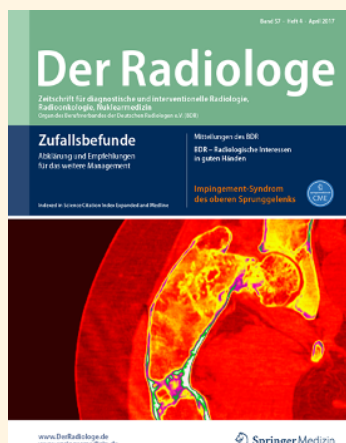

Für folgende Rubriken können Manuskripte eingereicht werden:

- Übersichten

- Originalien

- Quiz/Wie lautet Ihre Diagnose?

Um Ihnen bei der Manuskripterstellung behilflich zu sein, haben wir für unsere Autoren ausführliche Autorenleitfäden und Musterbeiträge für die verschiedenen Rubriken zusammengestellt.

Diese und weitere Hinweise zur Manuskripterstellung finden Sie online unter dem Menüpunkt „Hinweise für Autoren“ unter www.DerRadiologe.de.

Bitte reichen Sie Ihren fertigen Beitrag in elektronischer Form bei den zuständigen Schriftleitern ein:

\section{Übersichten/Originalien:}

Prof. Dr. Christian Herold, Wien

ute.woisetschlaeger@meduniwien.ac.at

\section{Quiz/Wie lautet Ihre Diagnose?}

Prof. Dr. Thomas Helmberger, München

thomas.helmberger@klinikum-muen-

chen.de

Sollten Sie noch Fragen zur Manuskriptgestaltung und zur Verwendung von DICOMMaterial haben, wenden Sie sich bitte an die Redaktion:

Claudia Zappe

claudia.zappe@springernature.com

Wir freuen uns auf Ihre Beiträge! Ihre Redaktion von Der Radiologe 